\title{
Sacral Insufficiency Fractures : How to Classify?
}

\author{
Gesa Bakker, M.D., ${ }^{1}$ Joerg Hattingen, M.D., ${ }^{2}$ Hartmut Stuetzer, M.D., ${ }^{3}$ Joerg Isenberg, M.D., Ph.D. ${ }^{4}$ \\ Hannover Region Public Health Department, Hannover Region, Hannover, Germany \\ Department for Radiology and Neuroradiology, ${ }^{2}$ KRH Nordstadt, Hannover, Germany \\ Institute for Medical Statistics, ${ }^{3}$ Computer Science and Epidemiology IMSIE, University of Cologne, Cologne, Germany \\ Department of Traumatology and Orthopedics, ${ }^{4}$ KRH Nordstadt, Hannover, Germany
}

Objective : The diagnosis of insufficiency fractures of the sacrum in an elder population increases annually. Fractures show very different morphology. We aimed to classify sacral insufficiency fractures according to the position of cortical break and possible need for intervention.

Methods : Between January 1, 2008 and December 31, 2014, all patients with a proven fracture of the sacrum following a low-energy or an even unnoticed trauma were prospectively registered : 117 females and 13 males. All patients had a computer tomography of the pelvic ring, two patients had a magnetic resonance imaging additionally : localization and involvement of the fracture lines into the sacroiliac joint, neural foramina or the spinal canal were identified.

Results : Patients were aged between 46 and 98 years (mean, 79.8 years). Seventy-seven patients had an unilateral fracture of the sacral ala, 41 bilateral ala fractures and 12 patients showed a fracture of the sacral corpus : a total of 171 fractures were analyzed. The first group $A$ included fractures of the sacral ala which were assessed to have no or less mechanical importance $(n=53)$ : fractures with no cortical disruption ("bone bruise") ( $A 1 ; n=2)$, cortical deformation of the anterior cortical bone $(A 2 ; n=4)$, and fracture of the anterolateral rim of ala ( $A 3 ; n=47)$. Complete fractures of the sacral ala $(B ; n=106)$ : parallel to the sacroiliac joint $(B 1 ; n=63)$, into the sacroiliac joint ( $B 2 ; n=19)$, and involvement of the sacral foramina respectively the spinal canal ( $B 3 ; n=24)$. Central fractures involving the sacral corpus $(C ; n=12)$ : fracture limited to the corpus or finishing into one ala $(C 1 ; n=3)$, unidirectional including the neural foramina or the spinal canal or both $(C 2 ; n=2)$, and horizontal fractures of the corpus with bilateral sagittal completion $(C 3 ; n=8)$. Sixty-eight fractures proceeded into the sacroiliac joint, 34 fractures showed an injury of foramina or canal.

Conclusion : The new classification allowes the differentiation of fractures of less mechanical importance and a risk assessment for possible polymethyl methacrylate leaks during sacroplasty in the direction of the neurological structures. In addition, identification of instable fractures in need for laminectomy and surgical stabilization is possible.

Key Words : Insufficiency fractures · Sacrum · Classification · Cementoplasty.

- Received : June 12, 2017 • Revised : August 7, 2017 • Accepted : August 18, 2017

- Address for reprints : Gesa Bakker, M.D.

Hannover Region Public Health Department, Weinstr. 2, Hannover 30171, Germany

Tel : +49-17660005294, Fax : +49-5116161123337, E-mail : gesabakker@gmx.de

This is an Open Access article distributed under the terms of the Creative Commons Attribution Non-Commercial License (http://creativecommons.org/licenses/by-nc/4.0) which permits unrestricted non-commercial use, distribution, and reproduction in any medium, provided the original work is properly cited. 


\section{INTRODUCTION}

The prevalence of insufficiency fractures of the sacrum increases accompanied with longer life expectancy in the last two decades $^{20,23,24)}$. Meanwhile lower back pain in elderly patients in combination with osteoporosis leads more often to a fracture of the sacral bone ${ }^{2)}$. Accurate diagnosis can often be delayed ${ }^{27)}$ since lower back pain has many, even competing, reasons. Moreover, the primary diagnostic using a pelvic anterior posterior X-ray overview may lead to a fracture of the anterior pelvic ring, but the sacral fracture and, in particular, the slightly displaced insufficiency fracture of the os sacrum is mostly not recognizable. While pain caused by a lesion of the anterior pelvic ring, subjected to less mechanical stress, shows a good controllability under moderate analgesia, insistent pain caused by the sacral fracture, often with a pain radiation down the dorsal thighs, remains. The pain can be provoked by pressure on the sacroiliac joint. Following clinical guidelines, computed tomography (CT) diagnostic is performed to detect the dimension of the sacral impact. Without treatment only few patients achieve sufficient mobility despite an extended treatment over several weeks ${ }^{2,27)}$. The in-hospital mortality as a consequence of uncontrolled pain and immobility caused by exacerbation of pre-existing comorbidities is about $10 \%$; even higher mortality is reported in the first year, ranging from 12 to $33 \%{ }^{2)}$. In a more vital Australian collective full independency and complete pain reduction is reported even after nine month ${ }^{11)}$. Nevertheless, conservative management with bed rest for 3 to 6 month is usually practiced, which might increase even up to 12 months in case of poor bone healing ${ }^{23)}$; this results in accelerated loss of bone mineral density. Costs caused by stationary treatment, surgical and rehabilitative therapy are considerable. As the awareness for this injury is constantly increasing ${ }^{4)}$, there is a distinct need for a morphological based convenient classification.

\section{MATERIALS AND METHODS}

Between January 1, 2008 and December 31, 2014, 130 consecutive (117 females and 13 males) patients with proven sacral fractures $(n=179)$ which had no or low energy trauma were prospectively examined. Ninety-seven underwent a sacroplasty (S1/S2 vertebroplasty) in local anesthesia. Five females had metachronous surgical stabilization of the ventral pelvic ring by plate osteosynthesis. In four females laminectomy and dorsal stabilization were urgently performed, 29 patients were treated conservatively.

Differentiating between left and right sacral ala involvement, 179 fractures were observed in the collective. Patients were identified in the emergency department when reporting a severe dull pain in the deeper lumbar spine, often with an aggravation while standing or walking and with no improvement under pain medication. Most patients complained about pain radiation to the dorsal thigh, most suffered from newly occurred immobility. A trauma was denied or not recalled, even pain often proceeded for several weeks. Occasionally a trivial movement like a stumble or a fall down on a chair relates with the beginning of complaints.

\section{Diagnostics and fracture classification}

Patients received a CT scan of the pelvic ring either in consequence of a ventral pelvic ring fractures in X-ray image or, in case of absence of ventral fracture, following severe pressure pain at the sacroiliac joint or of the dorsal os sacrum. In four cases CT was supplemented by magnetic resonance imaging (MRI). Bone bruise was seen in two of them. Images were analyzed in three axial sections from S1 to S3 and in coronal reconstructions. Fracture lines inside these three axial layers were summed up into one central section plane of the sacral bone and transferred into a schema. In the final step, fracture lines reaching the bones surface were pictured in a three-dimensional model with an anterior view on the sacral bone (not part of this analysis).

Group A included fractures of the sacral ala which were assessed to have no or less mechanical importance : fractures with no cortical disruption ("bone bruise") type A1, plastic deformation without an interruption of the anterior cortex and without visible interruption of cancellous bone type A2 and compression fractures of the anterolateral rim of ala type A3 (Fig. 1). Complete fractures of the sacral ala formed group B : fractures, starting in the anterior cortical bone, proceeded parallel to the sacroiliac joint type B2 and thirdly, when fracture lines were orientated medially, into neural foramina respectively the sacral canal type B3 (Fig. 2). By derogation from the ala fracture types a third group in the sacral corpus with a center of the fracture medial of the neural foramina was identified as group $\mathrm{C}$ : fracture lines starting in the anterior cortical bone 

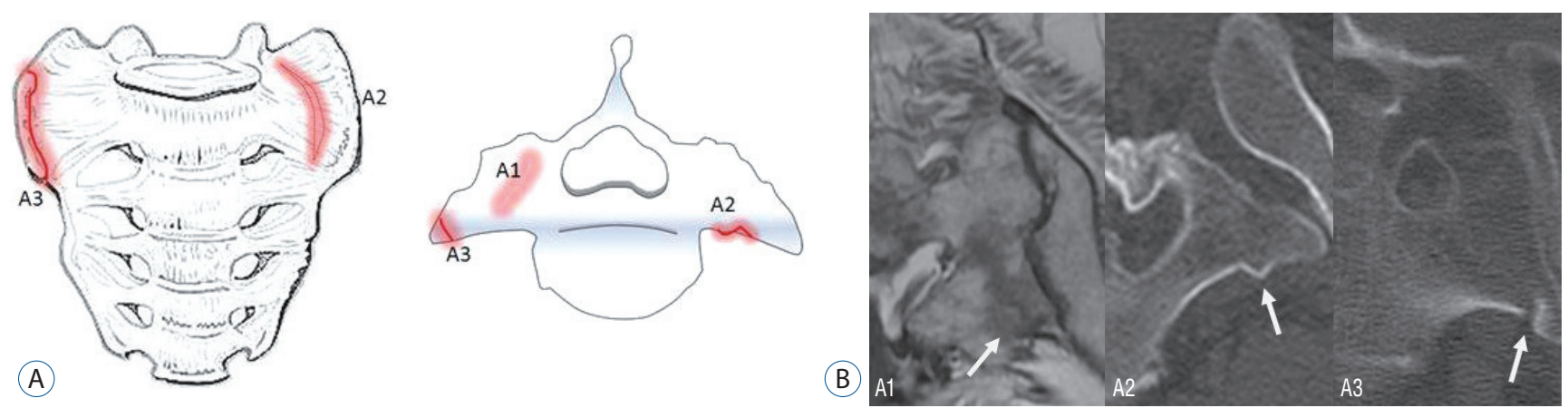

Fig. 1. A : Type A-fractures of the sacral ala : A1 with bone bruise (MRI) without a visible fracture line in the CT-scan; $A 2$ deformation of the anterior cortical bone without a cortical disruption; and $A 3$ anterolateral rim fracture of the ala with up to $1 \mathrm{~cm}$ distance in the direction of the medial sacroiliac joint. B : A1 with bone bruise in MRl; $\mathrm{A} 2$ plasticity of the cortical bone; $\mathrm{A} 3$ anterolateral fracture of the ala with up to $1 \mathrm{~cm}$ distance in the direction of the medial sacroiliac joint (rim fracture). A1 arrow shows bone bruise; A2 arrow shows plastic deformation of the anterior cortex; A3 arrow shows compression fracture of the anteolateral rim of ala.
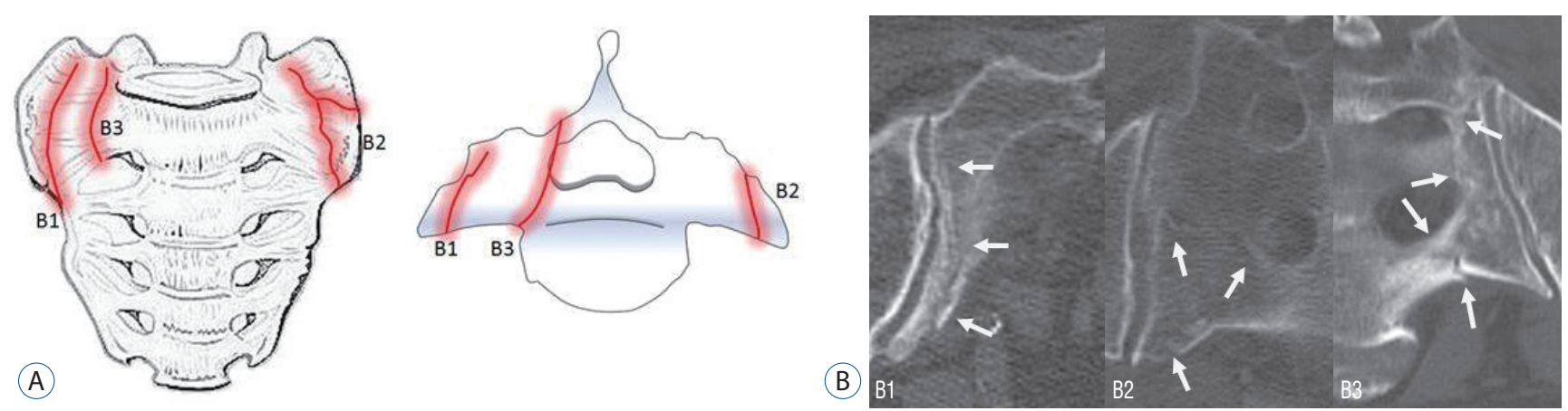

Fig. 2. A : Type B fractures of the sacral ala : $B 1$ fracture parallel to the sacroiliac joint; $B 2$ fracture involving the sacroiliac joint; and $B 3$ fracture with an involvement of the neural foramina or the spinal canal. B : B1 fracture parallel to the sacroiliac joint; $B 2$ fracture involving the sacroiliac joint; $B 3$ fracture with a participation of two neural foramina. B1 arrows show fracture following the sacroiliac joint; B2 arrows show the fracture process in the ala; $B 3$ arrows show the involvement of two neural foramina.
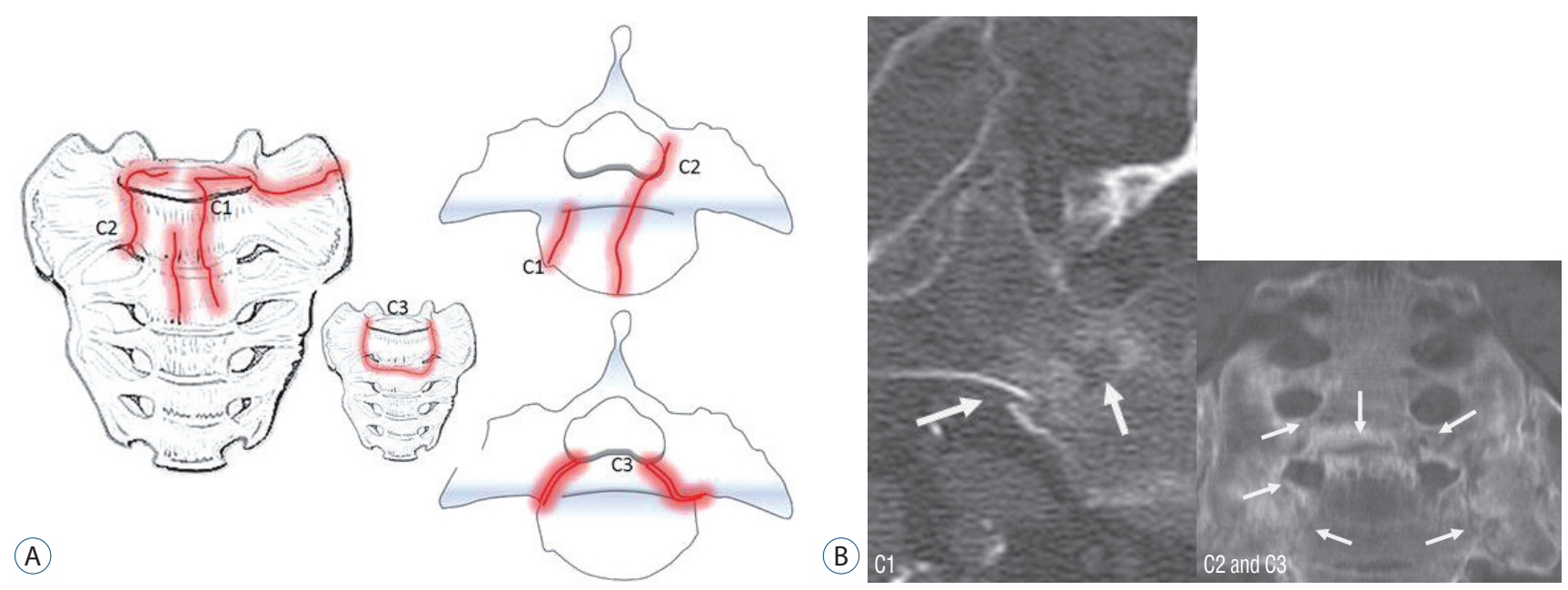

Fig. 3. A : Type C-or corpus-fractures : C1 fracture moves from anterior cortex dorsally or into the sacroiliac joint; $C 2$ fracture with an unilateral involvement of the neural foramina or the spinal canal; and C3 is unstable and represents bilaterally sagittal fractures combined with a transverse leasion. B: C1 fracture of the corpus with an extension in the anterior direction; $\mathrm{C} 2$ and $\mathrm{C} 3$ bilateral fracture of the corpus with an involvement of the neural foramina which are connected by a transverse fracture. C1 arrows show the fracture development from anterior to the dorsal cortex; $\mathrm{C} 2$ and $\mathrm{C} 3$ arrows show the bilateral fracture of the corpus, the arrow in the middle shows the fracture connection by a transverse component. 


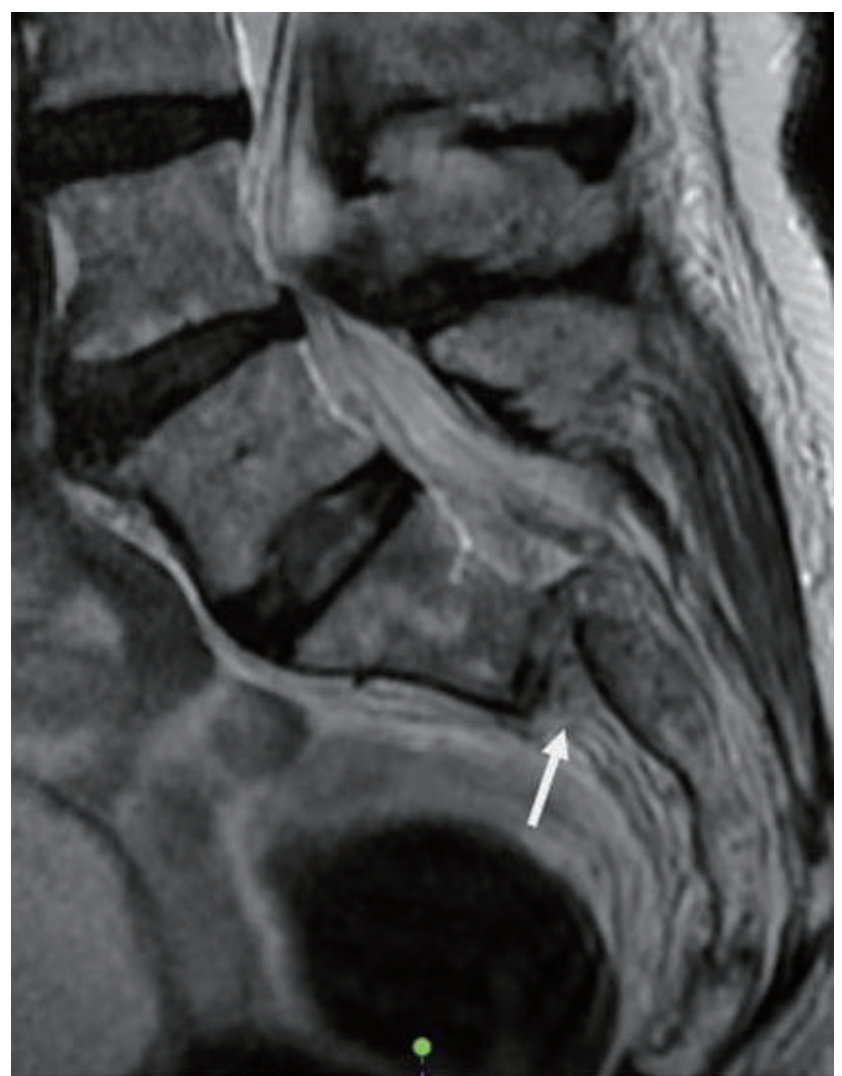

Fig. 4. Sagittal dislocation of type C3 fracture in magnetic resonance imaging. Arrow shows the horizontal dislocation by the transverse corpus fracture.

continued to the dorsal corpus respectively the dorsal iliosacral joint type $\mathrm{Cl}$, or into the neural foramina respectively the sacral canal of one side type $\mathrm{C}$, horizontal fractures of the corpus with bilateral sagittal completion type C3 (Figs. 3 and 4). Last type was defined as instable.

\section{RESULTS}

Patients age ranged from 46 to 98 years with a mean age 79.8 years for female patients $(\mathrm{n}=117)$ and 76.9 years for male patients $(n=13)$. Nine patients were younger than 60 years (all females), 26 patients were 90 years and older (all females). Analyzing impact of age, a continuous progress was seen up to the group 81 to 85 years (Fig. 5). Analyzing the age range, the number of cases increases (Fig. 6). Seventy-seven patients had a unilateral fracture of one sacral ala. There was no side preference : the right ala was affected 42 times, the left ala 35 . In 41 cases synchronous bilateral fractures occurred (Fig. 7). In 12 patients

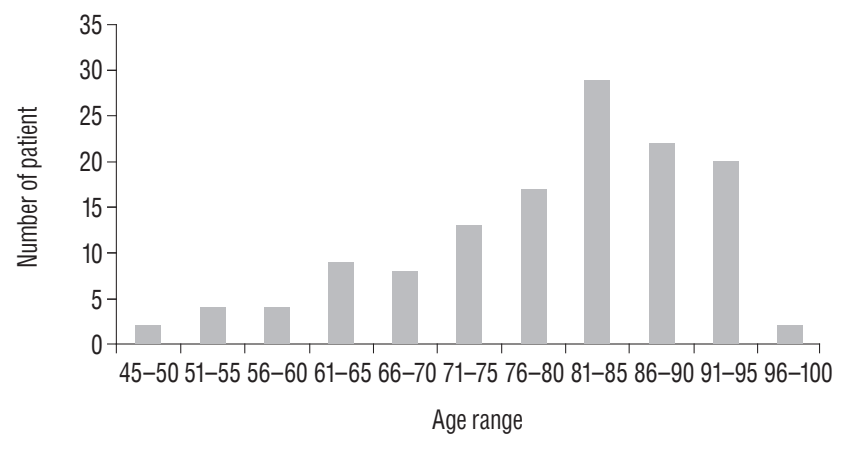

Fig. 5. Patient number in the distribution of age groups containing 117 females (average age, 79.8 years) and 13 males (average age, 76.9 years); average age of all of 79.5 years (range, 46 to 98 years).

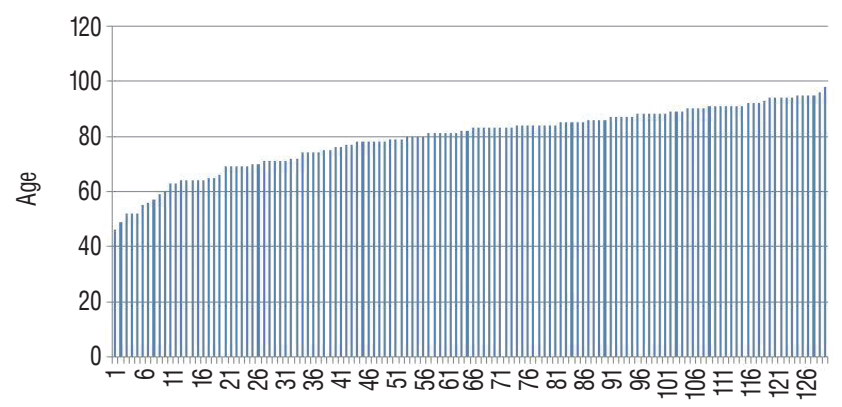

Fig. 6. Age range of 117 females (average age, 79.8 years) and 13 males (average age, 76.9 years) an average of all of 79.5 years (range, 46 to 99 ).

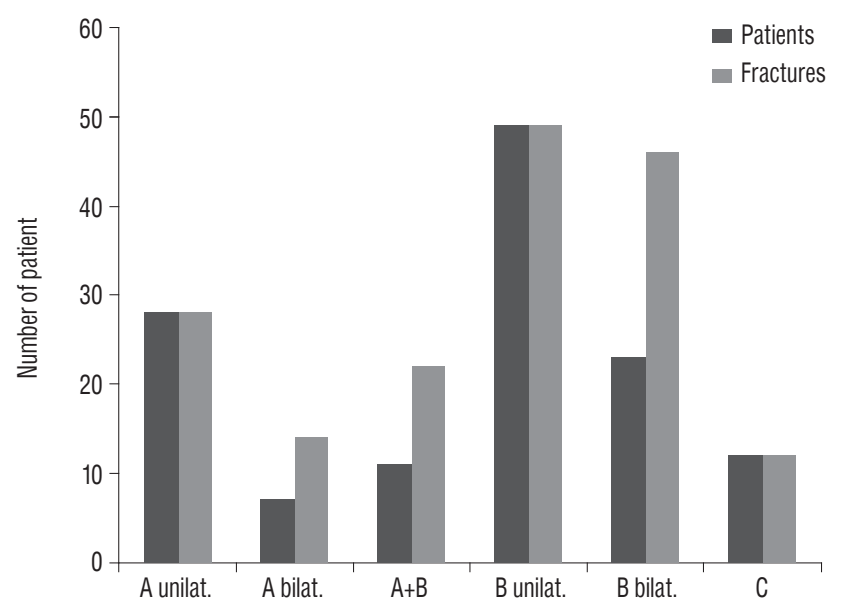

Fig. 7. Patient number and distribution of unilateral (unilat.) and bilateral (bilat.) ala fractures (types A and B) as well as of corpus fractures (type C).

the center of the fracture was localized central of the neural foramina in the corpus. 53 patients had a simultaneous participation of the anterior pelvic ring. Five of these underwent a surgical stabilization for pain control. According to radiological criteria, older and asymptomatic anterior fractures were found in 38 patients. 
Ala fractures without any mechanical significance summed up in group $A(n=53)$. The limitation of the diagnostic method caused an underrepresented proof of bone bruise without a cortical interruption (type Al; $\mathrm{n}=2$ ). The plastic deformation of osteoporotic cortical and cancellous bone was seen four times (type A2; $\mathrm{n}=4$ ). Compression fractures of the anterolateral rim of ala were quite usual (type A3; n=47; Figs. 1 and 8). Complete fractures of the ala occurred most frequently (group B; $\mathrm{n}=106$ ). Fractures exclusively parallel to the sacroiliac joint dominated

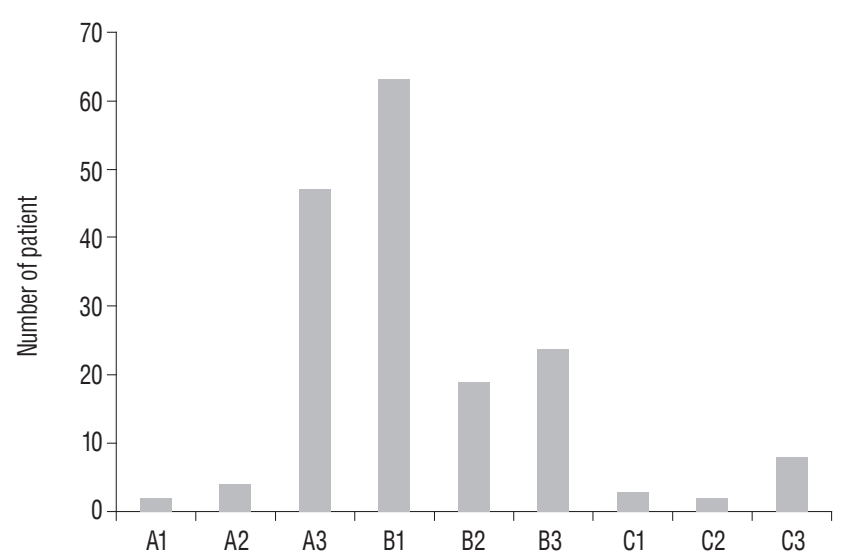

Fig. 8. The most frequent fracture was the fracture of the ala parallel to the sacroiliac joint (type B1) followed by the anterolateral ala rim fracture (type A3). There was an involvement of the spinal canal or the neural foramina in 34 cases (B3, C2, and C3). A corpus fracture was seen in 12 cases, thereof eight patients with a combination of transverse and bilateral sagittal fractures (type C3). (type $\mathrm{B} 1 ; \mathrm{n}=63$ ) in this group over fractures involving the sacroiliac joint (type B2; $\mathrm{n}=19$ ) or reaching into neural foramina respectively the sacral canal (type B3; $n=24$; Figs. 2 and 8). Sacral corpus fractures represented the smallest group (group C; $\mathrm{n}=12$ ). Involvement of the dorsal iliosacral joint (type $\mathrm{C} 1 ; \mathrm{n}=3$ ) or the neural foramina respectively the sacral canal of one side was rarely seen (type $\mathrm{C} 2 ; \mathrm{n}=2$ ). The bilateral spinopelvic dissociation dominated (type C3; n=8; Figs. 3, 4 and 8). Most fractures had no involvement of the neural foramina, the spinal canal or the sacroiliac joint : A1, A2, and B1 (n=69). Just as often there were fractures with a participation of the sacroiliac joint : $\mathrm{A} 3, \mathrm{~B} 2$, and $\mathrm{C} 1 \mathrm{n}=68)$. Fractures into the neural foramina and the spinal canal, which could cause passed by sacroplasty polymethyl methacrylate leakage, were seen less frequently ( $\mathrm{n}=34$; Figs. 8 and 9).

Four age range of patients were separated : 46 to 64 years, 65 to 74 years, 75 to 84 years and 85 to 98 years. In the first two groups fracture type A3, B1, and B3 were predominant. Simultaneously the instable fracture types were overrepresented. In the last two groups fracture type B3 receded into the background while type A3 and B1 dominated further on. Instable C3-fractures were rare. An explicit aberrance of the fracture type distribution between women and men was not found in our series.
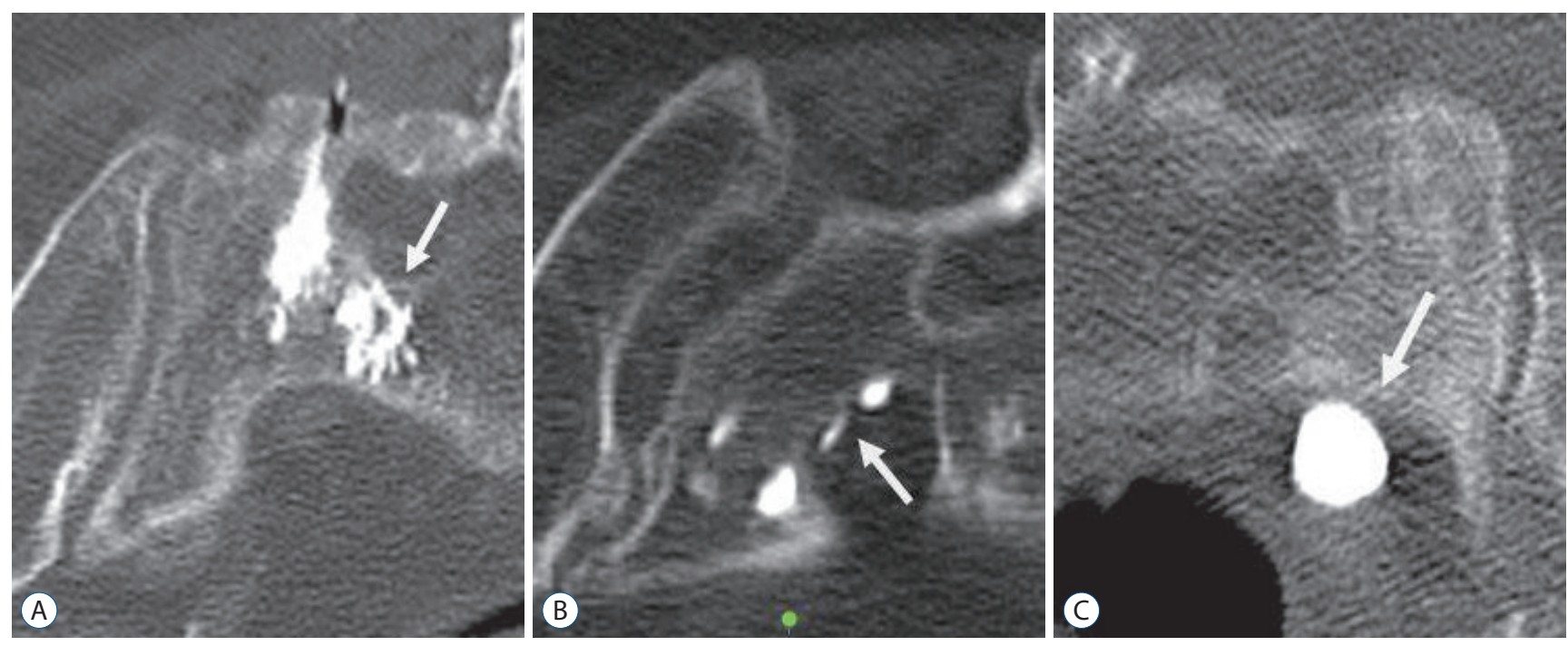

Fig. 9. A : Insertion of PMMA-cement near neurological structures does normally not cause a leakage through the cortical bone. B : In rare cases an uninjured dura seems to stop the leakage. Temporary neurological sensations had been reported. $C$ : If fractures reached the ventral border, a ventral passover was possible. Arrow in (A) shows PMMA-cement position inside the ala. Arrow in (B and C) show PMMA-leakage. PMMA : polymethyl methacrylate. 


\section{DISCUSSION}

Fatigue fractures occur if abnormal stress is applied to bone with a normal elastic resistance, whereas insufficiency fractures are caused by physiological stress applied to bone with deficient elastic resistance ${ }^{27)}$, when bone metabolism and remodeling capacity decrease according to age and diminished hormone production. Patients are old (mean 76 years up to 88 years) and predominantly females $(80 \% \text { to } 93 \%)^{1-3,6,7,9,10,18,19,21,22,25,31)}$. $78 \%$ of the patients show signs of osteoporosis, 22\% show osteopenia in dual energy X-ray absorptiometry combined with a substantial vitamin D deficiency ${ }^{2}$. Following Wolff's law, the resistibility of the bone is extenuated four times when bone density is bisected. The adaptation for mechanical stress extinguishes while repair-process still works. The reduction of trabecular bone outranges the reduction of the cortical bone. This explains why, in contrast to the non-osteoporotic post-traumatic fracture of the young, an involvement of the sacral foramina, respectively the sacral canal, is rare. Predisposing factors are primary and secondary osteopenia and osteoporosis (77.6\% and $22.4 \%$ ), often in combination with old age and female gender. Of importance are pelvic irradiation, prolonged corticoid treatment, rheumatoid arthritis, cushing disease, hyperparathyreodism, liver cirrhosis, osteomalacia and multiple myeloma ${ }^{2,11)}$. The stability of the elderly rigid sacroiliac ligaments prevails over the osteoporotic trabecular structure inside the sacral ala. The lateral part of the sacrum including the sacroiliac joint is protected by fracture by these strong ligaments, comparable to the pubic symphysis ${ }^{18)}$.

The pelvic ring is the strongest and largest osteo-ligament complex in the human body, it allows the repetitive vertical loading from the spine and upper body over the central sacrum to both acetabula. Sacral fractures in patients undergoing a high-energy trauma mean the separation of the key stone of the pelvic ring. They often are followed by hemodynamic instability and neurological impairment ${ }^{18)}$ that requires urgent surgical stabilization. Notwithstanding with the high-energy trauma, these fractures are only partially connected with fractures of the anterior pelvic ring. Compared to those, sacral insufficiency fractures arise from low-energy trauma, e.g., falling from a low position, chair or bed, or even stumble without a fall ${ }^{2,22)}$. For about half of the patients not remembering a suitable trauma, alternating weight bearing while walking followed by repetitive micro traumata to softened sacral ala might be dis- cussed. Probably the lack of anterior fractures is particularly often found among these patients. Unilateral stress loading for example through ankyloses of hip or sacroiliac joint of by hip endoprothesis seems to be associates with ipsilateral ala fractures $^{18)}$. Localization of insufficiency fracture and position of stress load while walking modeled biomechanically are congruent $^{18)}$. Cancellous density of the osteoporotic bone is reduced most of all just lateral to the neural foramina, whereas the anterior cortical bone of ala does not undergo excessive thinning as compared to other parts of os sacrum ${ }^{22)}$. These areas of greatest cancellous bone loss correspond to the locations of sacral insufficiency fractures according to our collective when corpus fractures were rarely seen.

The displacement in pelvic ring caused by high-energetic trauma is not seen in case of insufficiency fracture with at most mild misalignment that is clinically irrelevant; signs of mechanical instability are not found. Immobilizing pain, even in case of bone bruise respectively micro fracture without visible cortex fracture, dominates ${ }^{24)}$. Micro motion inside the fracture under alternating weight loading is interpreted as cause of pain ${ }^{4)}$.

Morphological descriptions based on CT-imaging and analyzing fracture and fissure localization, uni- and bilateral, with or without displacement, with sacroiliac or dorsal extension have been published ${ }^{22,24)}$, bilateral vertical fractures are leading $^{18,19)}$, usual both ala are affected ${ }^{27)}$. The horizontal fusion trough the corpus of two bilateral vertical ala fractures is interpreted as a progress of insufficiency by continued stress ${ }^{7,19,22}$, further characterized according to the degree of sagittal angulation $^{27)}$ and finally similar to spino-pelvic dissociation of jumper's fracture in the younger adult ${ }^{24)}$. Radionuclide bone scan show a characteristic $\mathrm{H}$ or butterfly shaped pattern of increased uptake ${ }^{9,10,12,31)}$. We also found this fracture form on CT images in eight of 130 patients (group C3). Simultaneous dorsal and anterior pelvic ring fractures in adults and elder patients are often a result of a fall on the side in up to $78 \%{ }^{1-3)}$. As in our collective a fall on the side was reported as an exception, less than half of the patients had a participation of the anterior pelvic ring.

In 1964, Judet et al. ${ }^{17)}$ published a classification system in order to define the pelvic approaches including those to transsacral fractures. In the classification system of pelvic ring fractures of Burgess and Tile respectively in the modified Tile AO Müller classification ${ }^{2,29)}$ direction of force transmission, range of instability, fractures and ligamental ruptures were conduct- 
ed : the transverse distal sacral fracture as a stable lesion of the dorsal arch is included in group A3 and their subtypes. The horizontal instability of B-type pelvic fractures includes also the incomplete disruption (type B1.2) and the incomplete compression in the sacral bone (type B2.1), furthermore, the complete instable C-type injuries with instable sacral disruption unilateral (type C1.3) and in both sides (type C2.3 and C3.3), too. The fractures morphology and localization are without significance, whereas a classification with a definition of the three zones transalar, transforaminal and central was introduced 1988 by Denis, furthermore, transverse, stable and unstable fractures and the rising risk of neurological lesion are differentiated $^{26)}$. The subdivision of Denis central fractures by Denis et al. ${ }^{8)}$ considers the horizontal translation in three types, and was complemented by Strange-Vognsen and Lebech ${ }^{28)}$. The lumbo-sacral involvement was added with three types by Isler $^{16)}$. These classifications of sacral fractures were developed in a younger adult collective, regularly by analyzing pelvic ring fractures : age ranged from 14 to 53 years, ISS 21 to 59 points, neurological lesion in more than $90 \%$, injuries of bladder, intestinum and genitalia in $63 \%{ }^{13,30)}$.

The latter classifications also used to classify insufficiency fractures in the past ${ }^{24)}$. In our opinion this is insufficient because the morphological aspect is not considered.

Rommens and Hofmann ${ }^{23)}$ published a classification of insufficiency fractures of the pelvic also including fractures of the sacrum analyzing pelvic fractures of 245 patients : isolated anterior or posterior pelvic fractures as well as their combinations were differentiated taking into account the degree of displacement and the resulting instability. Type IIa included nondisplaced isolated sacral fractures (unilateral or bilateral), type IIb a sacral crush and type IIc non-displaced sacral fractures, both with anterior fracture participation. Type III lesions were displaced, only type IIIc with an unilateral sacral fracture. Type $\mathrm{IVb}$ is characterized by a spinopelvic dissociation containing a horizontal fracture component. In contrast we saw no type III fractures through lack of displacing forces in the series of insufficiency fractures. Further the type IVa with fracture localization close to the sacroiliac joint was not observed, whereas type IVb met with our type C3. Type II (the undisplaced sacral fracture) corresponded to the fractures of our A and B types that we further differentiated into six subgroups with regard to the fractures localization. The relationship to the neural foramina or corpus was no content of Rommens publications. A comparison of the morphologic similar types IVb versus type C3 (18.4\% vs. 4.7\%) and type II versus type A/B (51.9\% vs. 92.9\%) suggests that different collectives were observed.

The origin of our classification arises from the sacroplasty and from fracture types. In our opinion other classification systems, e.g., the by Rommens et al. ${ }^{24)}$, do not fulfill those requirements because the morphological aspect is not considered. Their classification does not allow a closer view on endangered structures like the neural foramina. However, knowledge of the fracture lines within the os sacrum is necessary and important for sacroplasty indication.

It is generally accepted that intervention, if required, should be taken on a basis of a classification. In our collective, 94 patients received percutaneous sacroplasty in local anesthesia. In line with other study groups, sacroplasty remaines to be one of the most important treatment for the majority ${ }^{5,14,15)}$.

One complication seen in sacroplasty is the leakage of PMMA cement out of fracture lines, wherefore the morphologic relation of sacral fracture lines had to be taken into account related to the sacroiliac joint, the sacral foramina and the spinal canal. These complications were seen in our group in five cases with leakage into sacral foramina $(n=3)$ and through the anterior cortex $(n=2)$. As this complication was not detected in cases without visible cortical break highlights the importance of sufficient fracture line detection and classification.

Knowledge of the exact fracture lines also allows an adjustment of the PMMA cement, the selection of diameter of the canula and the position of the needle. Heo and Park ${ }^{15)}$ used a large diameter cannula and filler which is also used for vertebroplasty or osteoplasty to perform high viscosity, low pressure injections. Needle or canula location was important for foraminal leakage of PMMA. The tip of the canula being close to the foramen may increase the risk of foraminal PMMA leakage $^{15)}$. This may minimize or prevent severe PMMA complications.

Main parameters of the classification system were the cortical break and the fractures' morphology in the sacral bone. Fractures limited to the sacral ala were separated from the central C fractures (type A and B). In type A1 (only seen in MRI) and A2, with impaction and incomplete cortical break, PMMA cement leakage was impossible (or treatment not even required) and spontaneous healing probable. Fracture of the anterolateral rim of the ala, type A3, often had to be treated with sacroplasty; a limited leakage through the anterior cortex was 
imaginable. Further on, type B fractures represented complete fractures : type B1 was defined by an anterior cortical break as an option of leakage, type B2 with sacroiliac, and type B3 with intrathecal cortex break additionally. Finally the remaining corpus fractures were classified : type $\mathrm{C} 1$ without and type $\mathrm{C} 2$ with involvement of the cortical border to the neurological structures. The classification was concluding with the unstable type $\mathrm{C} 3$ fracture with two sagittal extensions involving the neural foramina and connected by a horizontal lesion that presented a higher risk of open reduction and fixation.

In contrast to the differentiated fracture classifications in young adult sacral insufficiency fractures in elderly-different in origin, bone quality, localization and morphology —are without a classification system regarding the special morphological aspects, especially if sacroplasty will be performed.

\section{CONCLUSION}

The new classification allows the differentiation of fractures of less mechanical importance and a risk assessment for possible PMMA leaks during sacroplasty in the direction of the neurological structures. In addition an identification of instable fractures in need for laminectomy and surgical stabilization is possible.

\section{CONFLICTS OF INTEREST}

The authors have no financial conflicts of interest.

\section{INFORMED CONSENT}

Informed consent was obtained from all individual participants included in this study.

\section{References}

1. Albertsen AM, Egund N, Jurik AG : Fatigue fracture of the sacral bone associated with septic arthritis of the symphysis pubis. Skeletal Radiol 24 : 605-607, 1995

2. Alnaib M, Waters S, Shanshal Y, Caplan N, Jones S, St Clair Gibson A, et al. : Combined pubic rami and sacral osteoporotic fractures: a prospective study. J Orthop Traumatol 13 : 97-103, 2012

3. Aretxabala I, Fraiz E, Pérez-Ruiz F, Ríos G, Calabozo M, Alonso-Ruiz A : Sacral insufficiency fractures. High association with pubic rami fractures. Clin Rheumatol 19 : 399-401, 2000

4. Bayley E, Srinivas S, Boszczyk BM : Clinical outcomes of sacroplasty in sacral insufficiency fractures: a review of the literature. Eur Spine J 18 : 1266-1271, 2009

5. Choi KC, Shin SH, Lee DC, Shim HK, Park CK : Effects of percutaneous sacroplasty on pain and morbility in sacral insufficiency fracture. J Korean Neurosurg Soc 60 : 60-66, 2017

6. Connolly LP, Drubach LA, Connolly SA, Treves ST : Young athletes with low back pain: skeletal scintigraphy of conditions other than pars interarticularis stress. Clin Nucl Med 29 : 689-693, 2004

7. Cooper KL, Beabout JW, Swee RG : Insufficiency fractures of the sacrum. Radiology 156 : 15-20, 1985

8. Denis F, Davis $\mathrm{S}$, Comfort $\mathrm{T}$ : Sacral fractures: an important problem. Retrospective analysis of 236 cases. Clin Orthop Relat Res 227 : 67-81, 1988

9. Finiels $H$, Finiels PJ, Jacquot JM, Strubel D : Fractures of the sacrum caused by bone insufficiency. Meta-analysis of 508 cases. Presse Med 26 : 1568-1573, 1997

10. Fujii M, Abe K, Hayashi K, Kosuda S, Yano F, Watanabe S, et al. : Honda sign and variants in patients suspected of having a sacral insufficiency fracture. Clin Nucl Med 30 : 165-169, 2005

11. Gotis-Graham I, McGuigan L, Diamond T, Portek I, Quinn R, Sturgess A, et al. : Sacral insufficiency fractures in the elderly. J Bone Joint Surg Br 76 : 882-886, 1994

12. Guilbeau JC, Arrivé L, Maruice F, Nahum H : Spontaneous fracture of the sacrum due to "insufficiency". An overlooked cause of low lumbago in elderly women. J Radiol 67 : 741-744, 1986

13. Hak DJ, Baran S, Stahel P : Sacral fractures: current strategies in diagnosis and management. Orthopedics 32 : 752-757, 2009

14. Hatgis J, Granville M, Jacobson RE, Berti A : Sacral insufficiency fractures: recognation and treatment in patients with current lumbar vertebral compression fractures. Cureus 9 : e1008, 2017

15. Heo DH, Park CK : Percutaneus sacroplasty for non-neoplastic osteoporotic sacral insufficiency fractures. Pain Physician 20 : 89-94, 2017

16. Isler $B$ : Lumbosacral lesions associated with pelvic ring injuries. J Orthop Trauma 4 : 1-6, 1990

17. Judet $R$, Judet J, Letournel $E$ : Fractures of the acetabulum: classification and surgical approaches for open reduction. Preliminary report. J Bone Joint Surg Am 46 : 1615-1646, 1964

18. Linstrom NJ, Heiserman JE, Kortman KE, Crawford NR, Baek S, Anderson $\mathrm{RL}$, et al. : Anatomical and biomechanical analyses of the unique and consistent locations of sacral insuffiency fractures. Spine (Phila Pa 1976) $34:$ 309-315, 2009

19. Longhino V, Bonora C, Sansone V : The management of sacral stress fractures: current concepts. Clin Cases Miner Bone Metab 8 : 19-23, 2011

20. Lourie $\mathrm{H}$ : Spontaneous osteoporotic fracture of the sacrum. An unrecognized syndrome of the elderly. JAMA 248 : 715-717, 1982

21. Majo NM, Helms CA : Sacral stress fractures in long-distance runners. 
AJR Am J Roentgenol 174 : 727-729, 2000

22. Richards AM, Coleman NW, Knight TA, Belkoff SM, Mears SC : Bone density and cortical thickness in normal, osteopenic, and osteoporotic sacra. J Osteoporos 2010 : 504078, 2010

23. Rommens PM, Hofmann A : Comprehensive classification of fragility fractures of the pelvic ring: recommendations for surgical treatment. Injury 44 : 1733-1744, 2013

24. Rommens PM, Wagner D, Hofmann A : Surgical management of osteoporotic pelvic fractures: a new challenge. Eur J Trauma Emerg Surg 38 : 499-509, 2012

25. Rousière $M$, Kahan $A$, Job-Deslandre $C$ : Postpartal sacral fracture without osteoporosis. Joint Bone Spine 68 : 71-73, 2001

26. Roy-Camille R, Saillant G, Gagna G, Mazel C : Transverse fracture of the upper sacrum. Suicidal jumper's fracture. Spine (Phila Pa 1976) 10 : 838-
845,1985

27. Schindler OS, Watura $R$, Cobby $M$ : Sacral insufficiency fractures. J Orthop Surg (Hong Kong) $15: 339-346,2007$

28. Strange-Vognsen $\mathrm{HH}$, Lebech $\mathrm{A}:$ An unusual type of fracture in the upper sacrum. J Orthop Trauma 5 : 200-203, 1991

29. Tile M : Acute pelvic fractures: I. Causation and classification. J Am Acad Orthop Surg 4 : 143-151, 1996

30. Tötterman A, Glott T, Madsen JE, Røise 0 : Unstable sacral fractures: associated injuries and morbidity at 1 year. Spine (Phila Pa 1976) 31 : E628-E635, 2006

31. Weber $M$, Hasler $P$, Gerber $H$ : Insufficiency fractures of the sacrum. Twenty cases and review of the literature. Spine (Phila Pa 1976) 18 : 25072512, 1993 